\title{
Polyarteritis nodosa presenting as a bladder outlet obstruction
}

\author{
M Borkum, ${ }^{1} \mathrm{MB}$ ChB, FCP (SA), MMed; H Y Abdelrahman, ${ }^{2} \mathrm{MSc}$, MRCP, MRCP (Rheum), Cert Rheum (SA); \\ R Roberts, ${ }^{3} \mathrm{MB}$ ChB, MMed, FCPath (SA) Anat; A A Kalla, ${ }^{2} \mathrm{MB}$ ChB, PhD; I G Okpechi, ${ }^{1}$ FWACP, PhD, Cert Nephrol (SA) Phys \\ ${ }^{1}$ Division of Nephrology and Hypertension, Faculty of Health Sciences, University of Cape Town, South Africa \\ ${ }^{2}$ Division of Rheumatology, Department of Medicine, Faculty of Health Sciences, University of Cape Town, South Africa \\ ${ }^{3}$ Division of Anatomical Pathology, Faculty of Health Sciences, University of Cape Town; and National Health Laboratory Service, \\ Cape Town, South Africa
}

Corresponding author: M Borkum (mborkum@gmail.com)

Polyarteritis nodosa (PAN) of the urinary tract is rare. An unusual case of systemic PAN involving the bladder neck is described. A 27-year-old man, with known diastolic hypertension diagnosed 2 years earlier, was admitted with chronic urinary obstruction complicated by hydronephrosis. He had symptoms of myalgia and weight loss, was afebrile but had an elevated erythrocyte sedimentation rate and acute-on-chronic renal impairment. All virological and serological tests including hepatitis B and anti-neutrophil cytoplasmic antibody were negative. A computed tomography scan of the brain revealed small-vessel disease. A bladder neck mass was visualised on cystoscopy. Histological examination of this demonstrated a medium-sized necrotising vasculitis with small-vessel fibrinoid necrosis suggestive of PAN. At least six of the American College of Rheumatology criteria for PAN were met. The patient was treated with pulses of intravenous cyclophosphamide and oral corticosteroids with a good clinical response.

S Afr Med J 2016;106(11):1086-1087. DOI:10.7196/SAMJ.2016.v106i11.11083

Polyarteritis nodosa (PAN) was first described by Kussmaul and Maier $^{[1]}$ in 1866 . The annual incidence is $<1 /$ million/year, while an incidence of $77 /$ million/year has been recorded in hepatitis B virus (HBV)-endemic areas, with a male/female ratio of $2-3: 1{ }^{[2]}$ The Chapel Hill Consensus Conference in 1994 defined PAN as 'necrotizing inflammation of medium sized or small arteries without glomerulonephritis or vasculitis in arterioles, capillaries or venules. ${ }^{[3]}$ PAN can be primary or secondary to viral infection (e.g. HBV) ${ }^{[4]}$ Antineutrophil cytoplasmic antibody (ANCA) testing is usually negative, which essentially differentiates it from microscopic polyangiitis. ${ }^{[4]}$ The disease often presents with vague and nonspecific signs and symptoms such as myalgia, arthralgia, fever, headaches, neuropathy and features of chronic kidney disease, and can manifest with symptoms of transient ischaemic attacks. ${ }^{[3]}$ If left untreated, PAN is usually fatal as a result of progressive renal failure or gastrointestinal complications. ${ }^{[3]}$

The urological manifestations of PAN documented in the literature include ureteric stenosis and orchitis. ${ }^{[5,6]}$ We present a case of systemic PAN involving the bladder in a man presenting with a bladder outlet obstruction due to a mass.

\section{Case report}

A 27-year-old man was admitted to a urology ward with chronic urinary obstruction and hydronephrosis diagnosed on an ultrasound scan. This was accompanied by severe myalgia, loss of weight (approximately $10 \mathrm{~kg}$ over 6 months) and suprapubic pain. His past history included only hypertension, diagnosed in 2010, which was never investigated for a possible secondary cause.

$\mathrm{He}$ was afebrile and a complete physical examination was unremarkable. Diastolic blood pressure readings were noted to be consistently elevated $(>90 \mathrm{mmHg})$.

Laboratory tests showed marked renal impairment (serum creatinine level $1096 \mu \mathrm{mol} / \mathrm{L}$ ), with a normocytic anaemia, an elevated erythrocyte sedimentation rate $(105 \mathrm{~mm} / \mathrm{h})$ and an elevated C-reactive protein level of $65 \mathrm{mg} / \mathrm{L}$. All virological and serological investigations, including HIV, hepatitis B surface antigen, hepatitis $\mathrm{C}$ virus antibodies, antinuclear antibodies, rheumatoid factor, a VDRL test, and perinuclear and cytoplasmic ANCA were negative. Complement levels ( $\mathrm{C} 3$ and $\mathrm{C} 4$ ) were normal. Further investigations including a chest radiograph, electrocardiogram and echocardiogram were all normal.

A cystoscopy and bladder neck resection were carried out for the urinary outlet obstruction. Histological examination of a specimen from the bladder neck revealed medium-vessel vasculitis suggestive of PAN (Fig. 1). An uncontrasted brain computed tomography (CT) scan revealed age-inappropriate atrophy with multiple lacunar infarcts due to significant small-vessel disease. CT angiography was not performed, as contrast media could not be used because of the patient's significantly impaired renal function.

The patient met 6 of the 10 American College of Rheumatology 1990 criteria for the classification of PAN, including weight loss $>4 \mathrm{~kg}$, diffuse myalgia, diastolic BP $>90 \mathrm{mmHg}$, elevated creatinine, appropriate histological findings and arteriographic abnormalities demonstrated on CT of the head. ${ }^{[7]}$ The Birmingham Vasculitis Activity Score (BVAS) was calculated at $13 \cdot{ }^{[8]}$

Oral prednisone $(1 \mathrm{mg} / \mathrm{kg})$ was commenced after excluding active and latent tuberculosis on sputum tests, chest radiography and Mantoux testing. Initial therapy with corticosteroids was, however, complicated by Klebsiella septicaemia, identified on blood culture and treated with appropriate antibiotics. On resolution of the sepsis, he was commenced on intravenous (IV) cyclophosphamide with mercaptoethane sulphonate $\mathrm{Na}$ (MESNA) according to the CYCLOPS protocol. ${ }^{[5]}$ The dose of cyclophosphamide given was $10 \mathrm{mg} / \mathrm{kg}$, reduced for renal function, every 2 weeks for 3 doses and then every 3 weeks for 


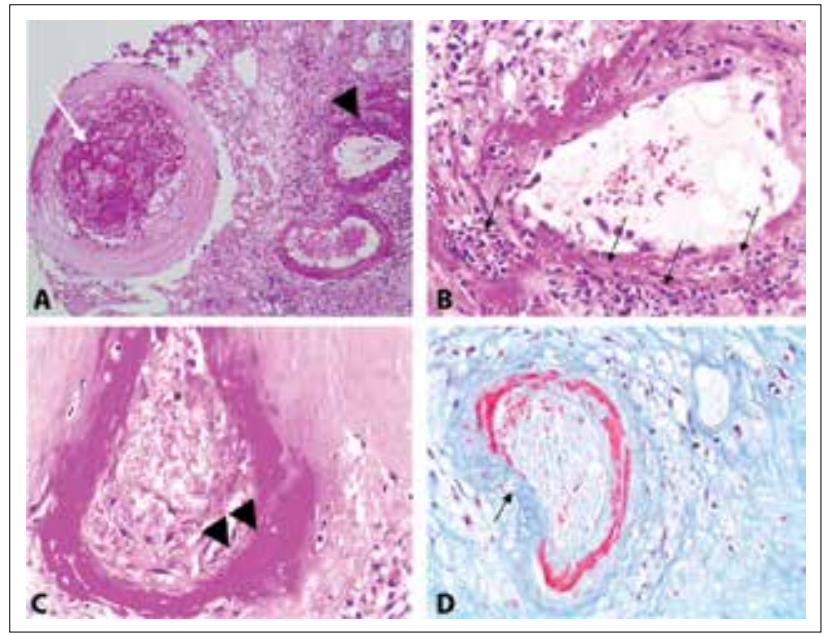

Fig. 1. (A) Sections of a medium-sized artery show luminal fibrin thrombi (white arrow). An adjacent small artery shows fibrinoid necrosis of the vessel wall (black arrowhead) (H\&E, 100 × magnification). (B) Sections of a medium-sized artery show transmural neutrophilic infiltrates and fibrinoid necrosis of the vessel wall (black arrows) (HÆE, 400× magnification). (C) Sections of a small artery show fibrinoid necrosis of the vessel wall (black arrowheads) (H\&E, 400× magnification). (D) Histochemical stain highlights fibrin deposition and fibrinoid necrosis of the vessel wall (black arrow) (Martius Scarlet Blue, 400× magnification).

3 doses. Oral prednisone was tapered after the first month by $5 \mathrm{mg}$ every 2 weeks down to a dose of $20 \mathrm{mg}$, then by $2.5 \mathrm{mg}$ monthly to a dose of $10 \mathrm{mg}$.

The patient responded well to treatment. His renal function improved without requiring dialysis and the urinary outlet obstruction resolved. He was discharged with follow-up visits at the rheumatology, renal and urology outpatient clinics. At 6-month follow-up the serum creatinine level was down to $526 \mu \mathrm{mol} / \mathrm{L}$ and his BVAS score was 2.

\section{Discussion}

This case reflects an unusual presentation of systemic PAN diagnosed incidentally on bladder neck resection specimens. Testicular involvement in systemic PAN is common, with a prevalence of up to $85 \%{ }^{[6]}$ We identified an isolated study in the literature, evaluating the radiographic findings of abdominal PAN, where extrarenal PAN (urethral, bladder) was found in 2 of the 7 patients studied. ${ }^{[5]}$ However, to our knowledge, PAN involving the urinary tract (especially the urethra and bladder neck) is not well described in the literature.

PAN is a potentially remitting disease with a lower relapse rate than granulomatosis with polyangiitis and microscopic polyangiitis. ${ }^{[3]}$ The symptoms of PAN can be nonspecific in the early stages. A high index of suspicion is therefore required to achieve an early diagnosis and prompt treatment with immunosuppressive therapy. While the most common cause of renal impairment is related to vasculitis of the renal vessels, this case demonstrates the unusual possibility of a 'postrenal' cause for renal failure in PAN.

The patient was treated successfully with pulses of IV cyclophosphamide. Compared with oral cyclophosphamide, this regimen offers the potential advantage of a lower cumulative dose and the likelihood of lower rates of adverse effects. ${ }^{[5]}$ Prolonged follow-up of the patient is needed to determine final outcomes.

1. Watts RA, Scott DGI. Polyarteritis nodosa. In: Watts RA, Scott DGI, eds. Vasculitis in Clinical Practice. London: Springer Verlag, 2010:95-106. DOI:10.1007/978-1-84996-247-6_10

2. Lhote F, Guillevin L. Polyarteritis nodosa, microscopic polyangitis and Churg-Strauss syndrome: Clinical aspects and treatment. Rheum Dis Clin North Am 1995;21(4):911-947.

3. Guillevin L, Lhote F. Polyarteritis nodosa and microscopic polyangitis. Clin Exp Immunol 1995;101(Suppl 1):22-23. DOI:10.1111/j.1365-2249.1995.tb06157.x

4. Sato O, Cohn DL. Polyarteritis and microscopic polyangiitis. In: Klippel JH, Dieppe PA, eds. Rheumatology. St Louis: Mosby, 2003.

5. Fraenkel-Rubin M, Ergas D, Sthoeger ZM. Limited polyarteritis nodosa of the male and female reproductive systems: Diagnostic and therapeutic approach. Ann Rheum Dis 2002;61(4):362-364. DOI:10.1136/ard.61.4.362

6. Jee KN, Ha HK, Lee IJ, et al. Radiologic finding of abdominal polyarteritis nodosa. AJR Am J Roentgenol 2000;174(6):1675-1679. DOI:10.2214/ajr.174.6.1741675

. Luqmani RA, Bacon PA, Moots RJ, et al. Birmingham vasculitis activity score (BVAS) in systemic necrotizing vasculitis. Q J Med 1994;87(11):671-678.

8. De Groot K, Harper L, Jayne DRW, et al. Pulse versus daily oral cyclophosphamide for induction Intern Med 2009:150(10):670-680. DOI-10.7326/0003-4819-150-10-200905190-0000

Accepted 30 June 2016. 